Fundación

Miguel Lillo

Tucumán

Argentina

\title{
Reproducción del gaviotín sudamericano Sterna hirundinacea (Charadriiformes: Laridae) en el Lago Epecuén, Buenos Aires, Argentina
}

\author{
Reproduction of the South American Tern Sterna hirundinacea \\ (Charadriiformes: Laridae) in Lake Epecuén, Buenos Aires, Argentina
}

Viviana Castro ${ }^{1 *}$, Juan José Abut ${ }^{1}$, Sebastián Preisz ${ }^{2}$, Laura Dodyk ${ }^{3}$, Candelaria Neyra 4 , Pablo Grilli 3,5,6

1 Servicio de Guardaparques de la Municipalidad de Adolfo Alsina, Av. Colón y Belgrano, (6430) Adolfo Alsina, Buenos Aires.

2 Yetapá Safaris Fotográficos, Calle 46 1974, (1900) La Plata, Buenos Aires.

3 Aves Argentinas / Asociación Ornitológica del Plata, Matheu 1245, (1249 AAB) Ciudad Autónoma de Buenos Aires.

4 Lavalle 173, (7540) Cnel. Suárez, Buenos Aires.

5 Dirección de Áreas Naturales Protegidas de la Provincia de Buenos Aires (OPDS), Av. 7, (1900) La Plata, Buenos Aires.

6 Cátedra de Ecología General y Recursos Naturales, Universidad Nacional Arturo Jauretche, Av. Calchaquí 6200, (1888) Florencio Varela, Buenos Aires.

*Correo electrónico: parinacota@hotmail.com

\section{RESUMEN}

El Gaviotín Sudamericano (Sterna hirundinacea) es una especie costero-marina, migratoria y exclusiva de Sudamérica. Si bien se ha registrado en varios cuerpos de agua interiores en territorio argentino, hasta el momento todos los eventos de nidificación se dieron sobre la costa marina, donde la abundancia de alimento (basada en Anchoita Argentina Engraulis anchoita) puede sostener colonias, a excepción de una pequeña colonia en el Río Negro. En febrero de 2020 se registraron adultos de Gaviotín Sudamericano alimentando con langostas a volantones, en el entorno del Lago Epecuén, en la Provincia de Buenos Aires. En octubre de 2020 se encontraron 126 nidos en una colonia sobre un islote con uno o dos huevos (promedio 1,7), pichones pequeños y huevos depredados. En época de cría, alimenta a sus pichones con una dieta compuesta en más del $90 \%$ de peces. Se observaron adultos cazando insectos y alimentando volantones en el aire. El Lago Epecuén es hipersalino, y

Ref. bibliográfica: V. Castro, J. J. Abut, S. Preisz, L. Dodyk, C. Neyra, P. Grilli. 2021. “Reproducción del gaviotín sudamericano Sterna hirundinacea (Charadriiformes: Laridae) en el Lago Epecuén, Buenos Aires, Argentina". Acta zoológica lilloana 65 (2): 173-181. doi: https://doi.org/10.30550/j.azl/2021.65.2/2021-06-10 - Recibido: 17 de marzo 2021 - Aceptado: 10 de junio 2021.

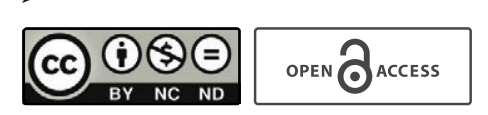

> URL de la revista: http://actazoologica.lillo.org.ar

- Esta obra está bajo una Licencia Creative Commons Atribución - No Comercial - Sin Obra Derivada 4.0 Internacional. 
no cuenta con peces, por lo que probablemente los insectos sean su reemplazo. El Gaviotín Sudamericano se reproduce siguiendo dos fenologías: el invierno austral (para Brasil y Uruguay) y el verano austral en la Patagonia Argentina. De acuerdo a su ubicación y al periodo de actividad, esta nueva colonia podría corresponderse con la fenología patagónica.

Palabras clave - Nidificación, colonia de cría, Encadenadas del Oeste.

\section{ABSTRACT}

The South American Tern (Sterna hirundinacea) is a coastal-marine species, migratory and exclusive to South America. Although it has been recorded in several inland water bodies in Argentine territory, so far, all nesting events have occurred on the marine coast, where the abundance of food (based on Argentine Anchoita Engraulis anchoita) can sustain colonies, except for a small colony on the Rio Negro. In February 2020, adults of South American Terns were registered feeding fledglings with lobsters, in the surroundings of Lake Epecuén, in the Province of Buenos Aires. In October 2020, 126 nests were found in a colony on an islet with one or two eggs (average 1.7), small chicks and predated eggs. During the breeding season, it feeds its chicks with a diet made up of more than $90 \%$ fish. Adults were observed hunting insects and feeding fledglings in the air. Lake Epecuén is hypersaline, and has no fish, so insects are probably its replacement. The South American Tern reproduces following two phenologies: the southern winter (for Brazil and Uruguay) and the southern summer in Patagonia Argentina. According to its location and the period of activity, this new colony could correspond to the Patagonian phenology.

Keywords - Nesting, breeding colony, Encadenadas del Oeste.

El Gaviotín Sudamericano (Sterna hirundinacea) es una especie de ave costero-marina, migratoria. Su distribución se restringe a Sudamérica, desde el sur de Brasil hasta la costa de Argentina (incluyendo las Islas Malvinas) por el Océano Atlántico, y a lo largo de las costas de Chile, Perú y Ecuador por el Océano Pacífico (Gochfeld y Burger, 1996).

En nuestro país la especie ha sido registrada en varios cuerpos de agua interiores, como el Embalse Potrerillos (Mendoza), y algunos lagos de la Patagonia, como el Lago Sarmiento (Chubut), Lago Gral. Carrera, Lago Argentino y Lago Viedma (Santa Cruz), en algunos puntos alejados de la costa marina (eBird, 2020; EcoRegistros, 2020). Pero hasta el momento, todos los eventos documentados de nidificación en colonias numerosas de la especie se sitúan en costas de mar tanto en islas como en el continente (Scolaro, Laurenti, Gallelli, 1996; Branco, 2003; Branco, 2004; Yorio, 2005; Alves Fracasso, Branco, Burger, Silveira, Verani, 2010; Hogan, Pellvitz, Vooren, 2010; Portflitt-Toro, et al., 2018), donde la abundancia de alimento puede sostener colonias (Branco, 2003). La única excepción está representada por el hallazgo de 11 
nidos sobre un islote en el Río Negro, a menos de $75 \mathrm{~km}$ de su desembocadura en la costa atlántica (Petracci y Pérez, 1997).

El 20 de septiembre, 11 de octubre y 29 de diciembre de 2019, VC y JJA registraron individuos adultos de Gaviotín Sudamericano en diferentes sitios alrededor del Lago Epecuén (377'59’'S, 6252’4'O), Partido de Adolfo Alsina, en la Provincia de Buenos Aires. El 3 de febrero de 2020, se recorrieron estos sitios constatando la presencia tanto de adultos como de volantones de Gaviotín Sudamericano. Cada vez que se observaron volantones, se verificó que éstos eran alimentados por los adultos. Coincidiendo con lo observado por Fernández Ajó, et al. (2011), los volantones eran alimentados por los adultos de a uno por vez, y en sus viajes, cada adulto acarreaba una única presa en el pico. Esta conducta fue observada tanto en vuelo (figura 1), so-
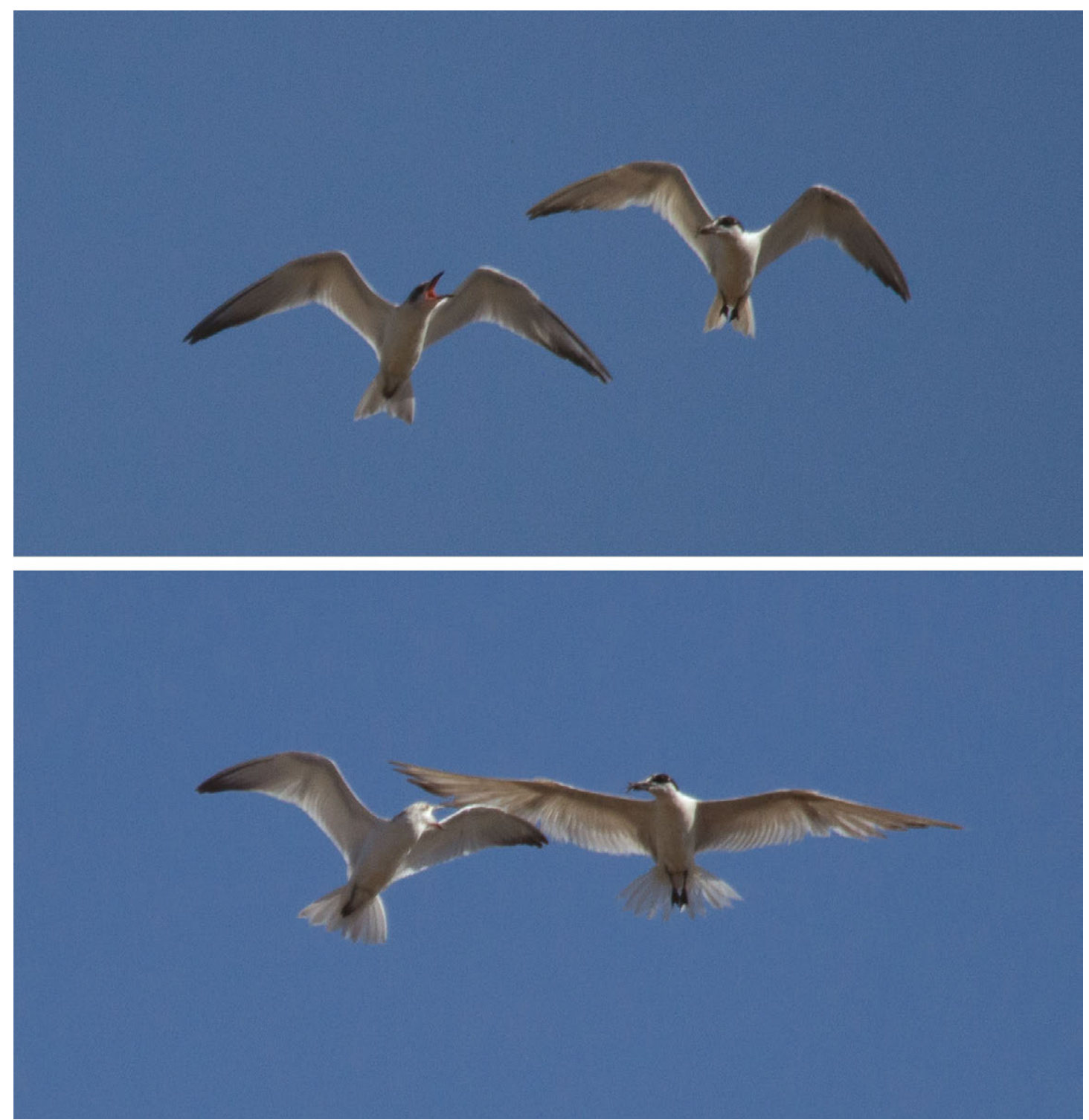

Figura 1: Volantón (siempre a la izquierda) y adulto (siempre a la derecha) acarreando una presa para alimentarlo en el aire, sobre un islote del Lago Epecuén (punto 2 de la tabla 1). Fotos: Pablo Grilli.

Figure 1: Fledgling (always on the left) and adult (always on the right) carrying a prey to feed him in the air, on an islet of Lake Epecuén (point 2 of table 1). Photos: Pablo Grilli. 
bre un islote del lago, como sobre la superficie de una charca temporal (figura 2). En cercanías de la Estación A. Vatteone, pudo verse a dos adultos capturando langostas sobre un pastizal, siguiendo un patrón que consistía en un vuelo corto y rectilíneo
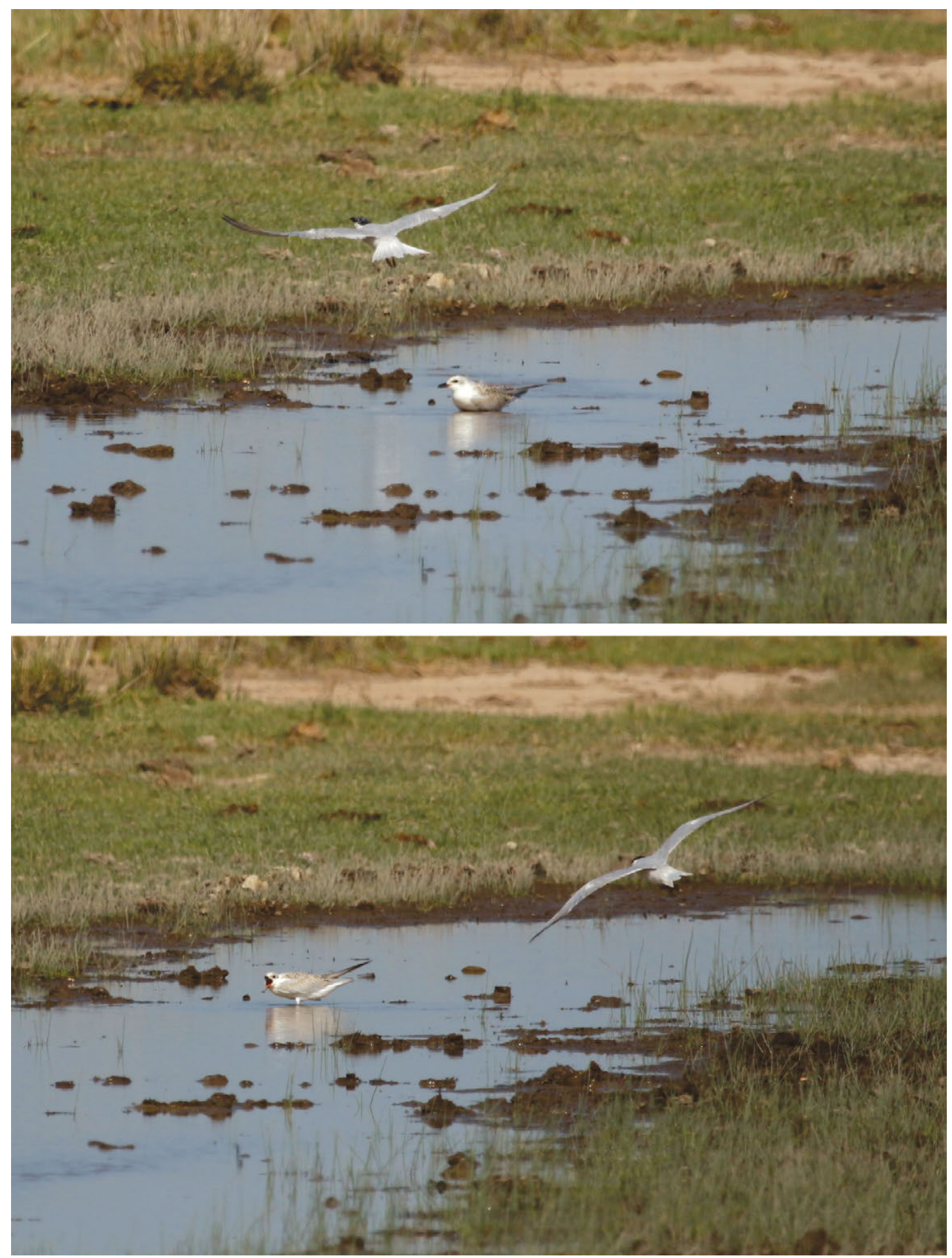

Figura 2: Volantón (siempre posado) y adulto (siempre en vuelo) en el momento en que lo alimentaba (punto 5 de la tabla 1). Fotos: Pablo Grilli.

Figure 2: Fledgling (always perched) and adult (always in flight) at the time of feeding (point 5 of table 1). Photos: Pablo Grilli. 
en contra del viento, a una altura de entre 5 y 7 metros sobre el suelo, para pasar a un aleteo rápido del tipo "halconeo" y luego una picada en vuelo rápido y plano, cerca de la superficie, para atrapar al insecto (figura 3). En la tabla 1 se expone un resumen de los hallazgos, que pueden ser referenciados en la figura 4.

Durante la época de cría, el Gaviotín Sudamericano alimenta a sus pichones con una dieta compuesta por peces en más del 90\% (Fernández Ajó, Gatto, Yorio, 2011). El Lago Epecuén (IBA AR267; BirdLife International, 2021) es un lago ubicado a una altura de más de $100 \mathrm{msnm}$., hipersalino, de unas 40.000 ha., y los únicos animales que habitan sus aguas son Artemia salina y aves acuáticas (Corti, 1925). Por lo tanto, la alimentación de los adultos y de los volantones de Gaviotín Sudamericano en este sitio no pudo estar basada en peces, y probablemente los insectos sean su reemplazo.

Dado que no pudo constatarse la presencia de nidos de la especie en esta primera instancia, VC y JJA llevaron a cabo una búsqueda intensiva de eventos de nidificación en el Lago Epecuén a partir del 11 de octubre de 2020, con una frecuencia semanal. El 22 de noviembre de 2020 se registraron por primera vez 126 nidos de Gaviotín Sudamericano formando una colonia en un pequeño islote de $460 \times 110$ m ubicado cerca del margen sur del lago (37 9'47.14"S, 6250’38.39'O). Los nidos contenían $1 \mathrm{o}$, mayormente, 2 huevos (1,7 promedio) y en esa primera visita se en-
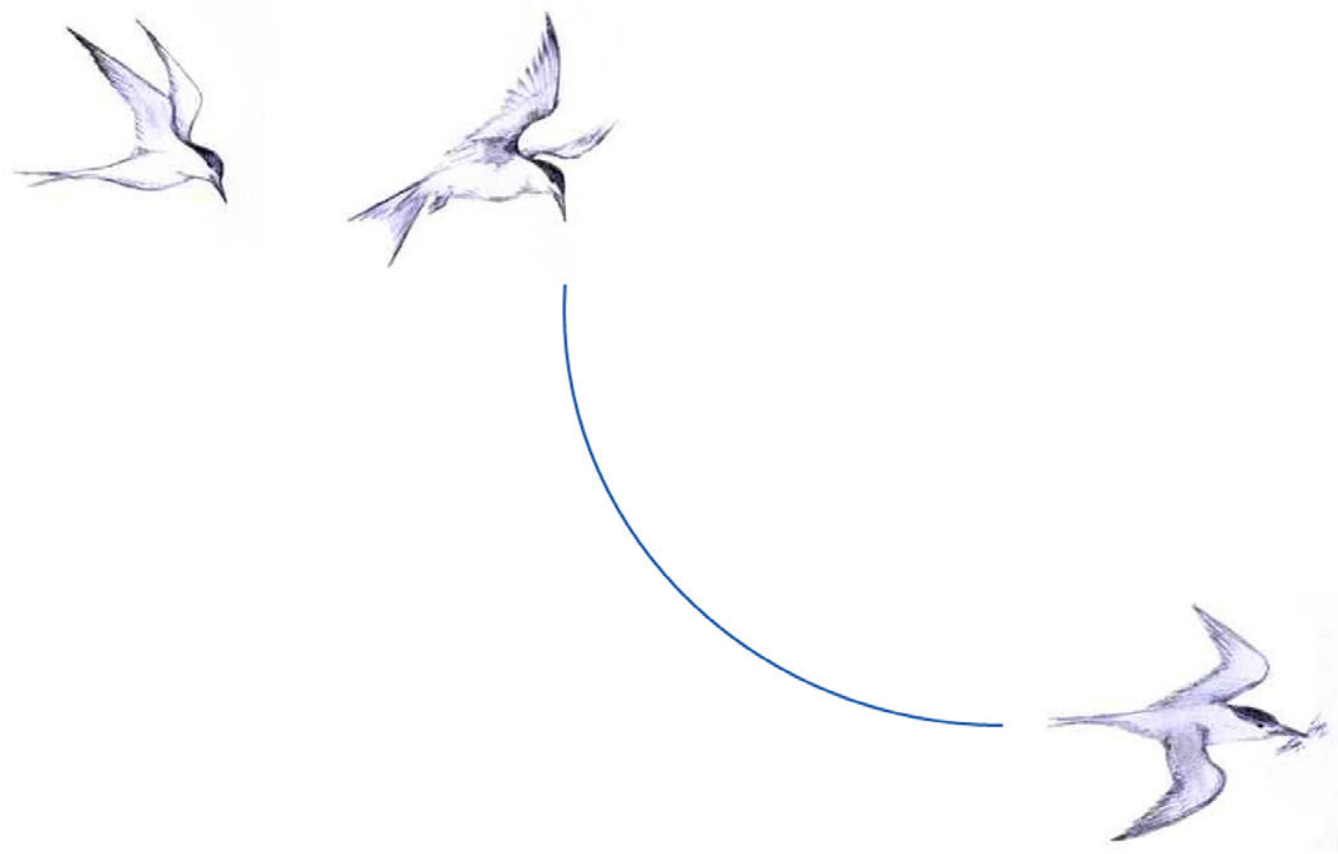

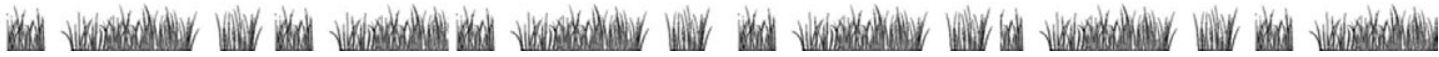

Figura 3: Esquema que expone la secuencia de movimientos que realizaron los adultos observados en el punto 4 (ver tabla 1) al momento de cazar langostas sobre un pastizal: 1) vuelo corto y rectilíneo en contra del viento; 2) aleteo rápido del tipo "halconeo"; 3) picada y vuelo rápido y plano, cerca de la superficie.

Figure 3: Scheme showing the sequence of movements carried out by the adults observed in point 4 (see table 1) when hunting lobsters on a grassland: 1) short and rectilinear flight against the wind; 2) rapid flutter of the "hovering" type; 3 ) dive and flight fast and flat, close to the surface. 
Tabla 1: Se describen las principales observaciones de la especie en el entorno del Lago Epecuén, entre septiembre de 2019 y febrero de 2020. A: adultos; V: volantones. La nomenclatura de los puntos es la misma que se emplea en la figura 4.

Table 1: Description of the main observations of the species in the surroundings of Lake Epecuén, between September 2019 and February 2020. A: adults; V: fledglings. The nomenclature of the points is the same as in figure 4.

\begin{tabular}{|c|c|c|c|c|}
\hline Puntos & Sep. & Oct. & Dic. & Feb. \\
\hline 1: Dormidero & $2 \mathrm{~A}$ & $2 \mathrm{~A}$ & 0 & 0 \\
\hline 2: Islote & 0 & $2 \mathrm{~A}$ & $5 A$ & $\begin{array}{l}5 \mathrm{~A} \text { y } 3 \mathrm{~V} \text {. Pudo registrarse la alimentación de } \\
\text { volantones en el aire }\end{array}$ \\
\hline 3: RP60 & 0 & 0 & 0 & $1 \mathrm{~A}$ en vuelo \\
\hline 4: Estación A. Vatteone & $8 \mathrm{~A}$ & $6 \mathrm{~A}$ & $5 A$ & $5 \mathrm{~A}$ alimentándose de langostas \\
\hline 5: Charca temporal & $6 \mathrm{~A}$ & $4 \mathrm{~A}$ & $4 \mathrm{~A}$ & $\begin{array}{l}\text { 2A y } 2 \mathrm{~V} \text {. Uno de los volantones permanecía } \\
\text { posado sobre el agua y era alimentado por dos } \\
\text { adultos }\end{array}$ \\
\hline Total de individuos & 16 & 14 & 14 & 18 \\
\hline
\end{tabular}

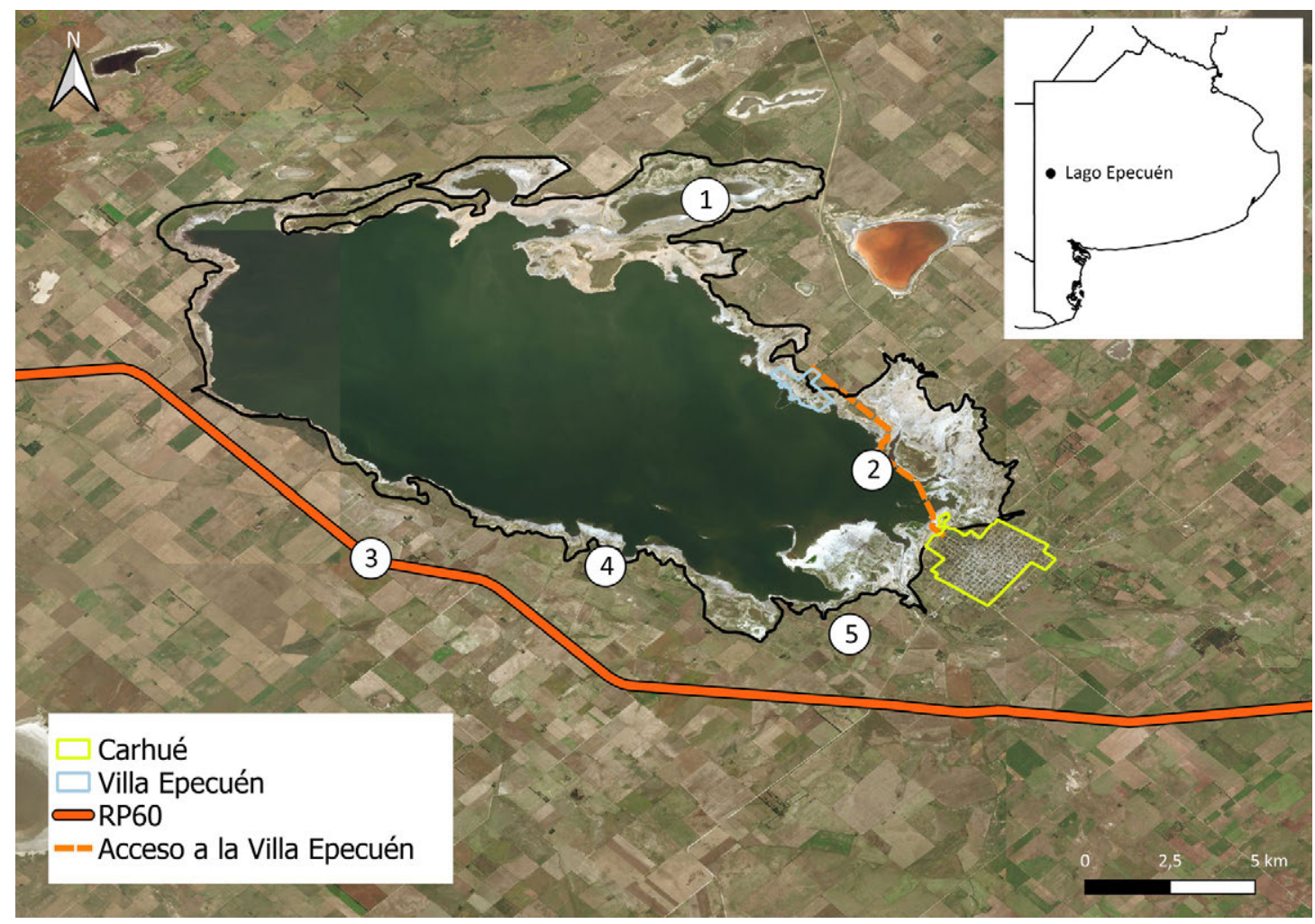

Figura 4: Mapa que muestra la ubicación de los puntos en el entorno del Lago Epecuén que se describen en la tabla 1 (adaptado de Google Earth, 2020).

Figure 4: Map showing the location of the points, in the surroundings of Lake Epecuén, described in table 1 (adapted from Google Earth, 2020).

contraron 8 pichones pequeños y se hallaron 5 huevos depredados (figura 5). En una segunda visita al islote, el 28 de noviembre, el número de pichones ascendía a 66.

Según Faria, et al. (2010), la reproducción de las poblaciones del Océano Atlántico del Gaviotín Sudamericano sigue un modelo particular, con dos fenologías distintas: el invierno austral para colonias de la costa brasileña y uruguaya (abril- 

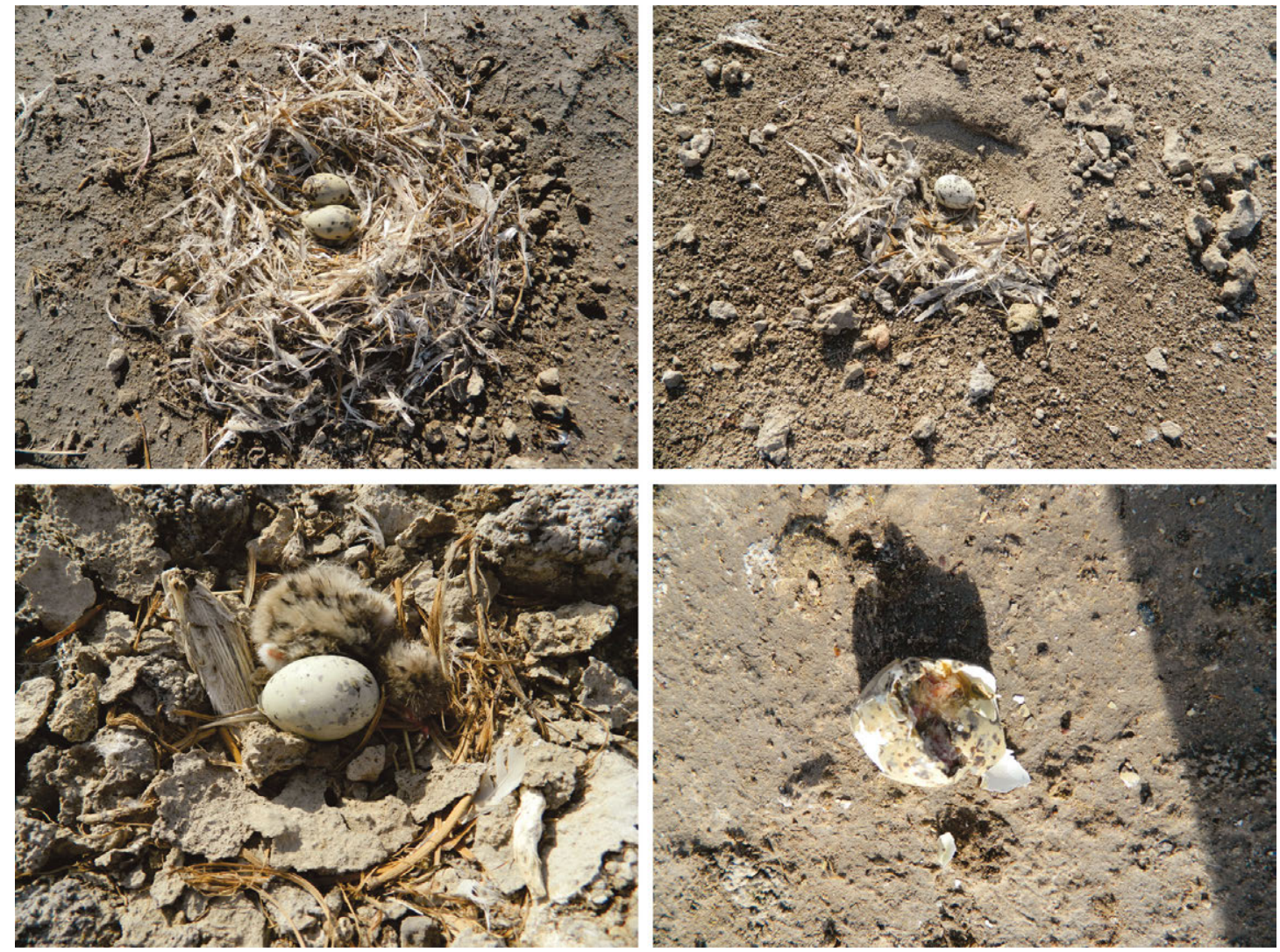

Figura 5: Nidos con dos y un huevo (arriba, izquierda y derecha, respectivamente) de Gaviotín Sudamericano, pichón en nido y huevo depredado (abajo, izquierda y derecha, respectivamente). Fotos: Juan José Abut.

Figure 5: Nests with two and one egg (top, left and right, respectively) of South American Tern, nestling in nest and preyed egg (bottom, left and right, respectively). Photos: Juan José Abut.

septiembre; Branco, 2003; Campos, Paludo, Faria, Martuscelli, 2004) y el verano austral en la Patagonia Argentina (noviembre-enero; Scolaro, et al., 1996). De acuerdo a su ubicación y al periodo de actividad, esta nueva colonia descubierta podría corresponderse con la fenología patagónica.

La dieta del Gaviotín Sudamericano fue estudiada a lo largo de distintos puntos de la costa atlántica, donde se alimenta principalmente de Anchoíta Argentina (Engraulis anchoita), tanto durante el período de invernada como durante el período reproductivo, seguida en orden de importancia por otras especies de peces, crustáceos e insectos (Favero, Bó, Silva Rodríguez, García Mata, 2000; Alfaro, Mauco, Norbis, Lima, 2011; Fernández Ajó, et al., 2011; Mariano Jelicich, et al., 2011; Woehler, Saviolli, Francini, Neves, Francini, 2013; Gatto y Yorio, 2016). Si bien el Lago Epecuén forma parte del sistema de Lagunas Encadenadas del Oeste, y se ubica a menos de $15 \mathrm{~km}$ de otros cuerpos de agua de importancia donde estas aves podrían capturar peces, la observación de individuos adultos cazando langostas y alimentando con ellas a volantones (ver figura 1) indicaría la importancia de los insectos en la dieta durante el periodo reproductivo para esta colonia de la especie. Anteriormente se habían registrado Ortópteros durante el periodo reproductivo de la especie en Brasil (Woehler, et al., 2013) y Uruguay (Alfaro, et al., 2011). 
Éste es el primer hallazgo de reproducción del Gaviotín Sudamericano en un cuerpo de agua interior para la Argentina. La localidad de avistaje más cercana para la especie es Bahía Blanca (eBird, 2020; EcoRegistros, 2020; Abel Gofio com. pers., 11 de junio de 2020), a unos $170 \mathrm{kms}$, mientras que a unos $385 \mathrm{~km}$ se encuentra la Isla Jabalí, donde se ubica la colonia de nidificación conocida más cercana (Zalba y Belenguer, 1996; Suárez, Marinao, Kasinsky, Yorio, 2014).

\section{AGRADECIMIENTOS}

Agradecemos al Lic. Javier Andrés, intendente Municipal de Adolfo Alsina, por su colaboración, a Nicolás Pérez por la ayuda con las fotografías y a Abel Gofio por el aporte de su experiencia. Parte de este trabajo se desarrolló en el marco del Censo Nacional de Falaropos 2020.

\section{LITERATURA CITADA}

Alfaro, M., Mauco, L., Norbis, W., Lima, M. (2011). Temporal variation on the diet of the South American Tern (Sterna hirundinacea, Charadriiformes: Laridae) on its wintering grounds. Revista Chilena de Historia Natural, 84, 451-460.

Alves Fracasso, H. A., Branco, J. O., Burger, J., Silveira, L. F., Verani, J. R. (2010). Breeding biology of South American Tern from Cardos Island, Santa Catarina State, Brazil. Journal of Oceanography and Marine Science, 13, 53-63.

BirdLife International (2021). Important Bird Areas factsheet: Lagunas Encadenadas del Oeste de la Provincia de Buenos Aires. (Descargado de http://www.birdlife. org el $05 / 03 / 2021)$.

Branco, J. O. (2003). Reprodução de Sterna hirundinacea Lesson e S. eurygnatha Saunders (Aves, Laridae), no Litoral de Santa Catarina, Brasil. Revista Brasileira de Zoología, 20, 655-659.

Branco, J. O. (2004). Aves marinhas e insulares brasileiras: bioecologia e conservação. Editora da UNIVALI, Itajaí, Brazil.

Campos, F. P, Paludo, D., Faria, P. J. Martuscelli, P. (2004). Aves insulares marinhas, residentes e migratórias, do litoral do Estado de São Paulo. En Aves marinhas e insulares brasileiras: bioecologia e conservação (233-254). Editora da UNIVALI.

Corti, H. (1925). Laguna de Epecuén (Carhué), Provincia de Buenos Aires. República Argentina, Ministerio de Agricultura de la Nación. Dirección General de Minas, Geología e Hidrología.

EBird (2020). Charrán sudamericano (Sterna hirundinacea). Ficha de la especie. (Accedido de https://ebird.org/argentina/home el 17/03/2020).

EcoRegistros (2020). Gaviotín Sudamericano (Sterna hirundinacea). Ficha de la especie. (Accedido de http://www.ecoregistros.org el 17/03/2020).

Faria, P. J., Campos, F. P., Branco, J. O., Musso, C. M., Morgante, J. S., Bruford, M. W. (2010). Population structure in the South American tern Sterna hirundinacea in 
the South Atlantic: two populations with distinct breeding phenologies. Journal of Avian Biology, 41, 378-387.

Favero, M., Bó, M., Silva Rodríguez, M. P., García Mata, C. (2000). Food and feeding biology of the South American Tern during nonbreeding season. Waterbirds, 23, 125-129.

Fernández Ajó, A. A., Gatto A., Yorio, P. (2011). Patterns of prey provisioning in relation to chick age in the South American Tern (Sterna hirundinacea). Ornitología Neotropical, 22, 361-368.

Gatto, A., Yorio, P. (2016). Assessing the trophic niche of South American Terns integrating conventional and isotopic methods. Emu- Austral Ornithology, 116, 230-240.

Gochfeld, M., Burger, J. (1996). Family Sternidae. En Handbook of the birds of the world. Volume 3: Hoatzin to auks (624-667). Lynx Edicions.

Hogan, R. I., Pellvitz, L. J., Vooren, C. M. (2010). Breeding biology of South American Tern Sterna hirundinacea (Charadriiformes: Sternidae) on Deserta Island, southern Brazil. Revista Brasileira de Ornitologia, 18, 207-215.

Mariano Jelicich, R., Silva Rodriguez, M. P., Copello, S., Seco Pon, J. P., Berón, M. P., Mauco, L. (2011). The diet of the South American Tern: the Argentine Anchovy as key prey in the non-breeding season. Emu, 111, 292-296.

Petracci, P. F., Pérez, C. H. F. (1997). Primer registro de nidificación en ambiente fluvial (Río Negro, Argentina) del Gaviotín Sudamericano Sterna hirundinacea. El Hornero, 14, 267-268.

Portflitt-Toro, M., Miranda-Urbina, D., Fernández, C., Luna, N., Serratosa, J., Thiel, M., Luna-Jorquera, G. (2018). Breeding of the South American Tern (Sterna hirundinacea) on anchored boats in Conquimbo, Northern Chile. Ornitología Neotropical, 29(1), 187-191.

Scolaro, J. A., Laurenti, S., Gallelli, H. (1996). Nesting and breeding biology of the South American Tern in northern Patagonia. Journal of Field Ornithology, 67, 17-24.

Suárez, N., Marinao, C., Kasinsky, T., Yorio, P. (2014). Distribución reproductiva y abundancia de gaviotas y gaviotines en el Área Natural Protegida Bahía San Blas, Buenos Aires. El Hornero, 29, 29-36.

Woehler, E. J., Saviolli, J. Y., Francini, C. L. B., Neves, T., Francini, R. B. (2013). Insect prey of breeding South American terns. Marine Ornithology, 41, 199-200.

Yorio, P. (2005). Estado poblacional y de conservación de gaviotines y escúas que se reproducen en el Litoral Marítimo Argentino. El Hornero, 20, 75-93.

Zalba, S. M., Belenguer, C. J. (1996). Nidificación del Gaviotín Sudamericano (Sterna hirundinacea) en Bahía San Blas, Buenos Aires. Nuestras Aves, 35,33-34. 